\title{
Automated Image Forgery Detection through Classification of JPEG Ghosts
}

\author{
Fabian Zach, Christian Riess and Elli Angelopoulou \\ Pattern Recognition Lab \\ University of Erlangen-Nuremberg \\ $\{$ riess, elli\}@i5.cs.fau.de
}

\begin{abstract}
We present a method for automating the detection of the socalled JPEG ghosts. JPEG ghosts can be used for discriminating singleand double JPEG compression, which is a common cue for image manipulation detection. The JPEG ghost scheme is particularly well-suited for non-technical experts, but the manual search for such ghosts can be both tedious and error-prone. In this paper, we propose a method that automatically and efficiently discriminates single- and double-compressed regions based on the JPEG ghost principle. Experiments show that the detection results are highly competitive with state-of-the-art methods, for both, aligned and shifted JPEG grids in double-JPEG compression.
\end{abstract}

\section{Introduction}

The goal of blind image forensics is to determine the authenticity of an image without using an embedded security scheme. With the broad availability of digital images and tools for image editing, it becomes increasingly important to detect malicious manipulations. Consequently, image forensics has recently gained considerable attention. Most existing methods fall into two categories: a) detecting traces of a particular manipulation operation and b) verifying the "rationality" of expected image artifacts. Good surveys on such methods are [10, 4]. For instance, methods for copy-move forgery detection search for duplicated content within the same image (see e. g. [3]). However, traces from such a copying operation may be visible to the eye. If a manipulator is careful, he might hide such visible traces using post-processing operations. To counter a careful, yet not technically educated forger, a number of researchers focused on invisible cues for image manipulation. One of the most widely used invisible indicators are JPEG artifacts. Their use in image forgery detection is based on the following key observation: every time that a JPEG image is recompressed, the statistics of its compression coefficients slightly change.

Consider, for example, the case where a manipulator takes a JPEG image, alters part of the image, and saves it again as a JPEG image. Then, the (unaltered) background is compressed twice, while the repainted area appears to be compressed only once in JPEG format - because the JPEG artifacts of the initial compression were destroyed by the painting operation. 
Thus, a common goal in JPEG-based forensics is to detect areas with differing numbers of JPEG compression, and report such an irregularity. For instance, Lin et al. [7] showed how the use of different quantization matrices in the first and second compression leads to a telltale high frequency component in the coefficient spectrum. However, their method assumes that the JPEG block grids of the first and second compression are exactly aligned. This holds for image regions in the background, as in the previous example. In more general scenarios, so-called "shifted double-JPEG (SD-JPEG) compression" can be detected. For instance, Qu et al. [8] developed a method for handling arbitrary block grid alignments using independent component analysis. Barni et al. [1] proposed a method that purely relies on non-matching grids. All these methods assume different quantization matrices for the first and second compression. Huang et al. [6] showed how to detect recompression with the same quantization matrices by exploiting numerical imprecisions of the JPEG encoder.

While the majority of the presented approaches rely on statistics, Farid [5] recently presented a perception-oriented SD-JPEG method, called "JPEG ghost" detection. It assumes that the first compression step was conducted on a lower quality level than the second step. Then, it suffices to recompress the image during analysis with various lower quality levels. Difference images are subsequently created by subtracting the original image from the recompressed versions (see Sec. 2). For approximately correct recompression parameters, the double compressed region appears as a dark "ghost" in the difference image.

The simplicity of this approach has several advantages: it is easy to implement, its validity can be simply explained, visually verified and demonstrated to non-technical experts. However, an important drawback is the lack of automation for JPEG ghost detection. It is currently infeasible for a human expert to visually examine the difference images for all possible parameters.

The goal of this paper is to address this drawback. We present a method that fully automates the detection of JPEG ghosts. A human expert can still make use of the JPEG ghost scheme, but is relieved from the requirement of manually analyzing hundreds of images. We designed 6 features that operate on the difference images to distinguish between single and double-compressed regions in the JPEG images. A comparison to the method of Lin et al. [7], as well as to results reported by other authors, shows that our JPEG ghost detection has very competitive performance.

\section{JPEG Ghost Observation}

We briefly restate Farid's ghost observation [5]. Let $I_{q_{1}}$ be an input image that has been compressed with JPEG quality $q_{1}$. Assume that a region of the image has been previously compressed with JPEG quality $q_{0}$, where $q_{0}<q_{1}$. To detect this doubly compressed region, define a set of quality factors $Q=\left\{q_{2} \mid 0<q_{2}<\right.$ $\left.q_{1}\right\}$. Recompressing image $I_{q_{1}}$ with the factors in $Q$ yields a set of test images 

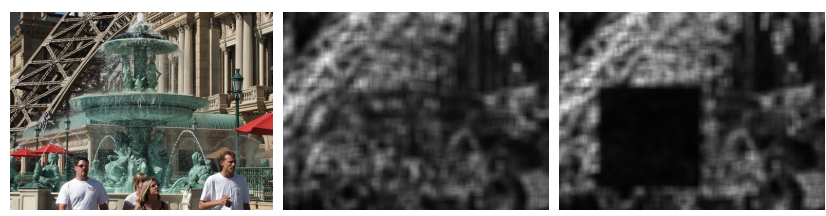

Fig. 1. Example JPEG ghost. Left: a rectangular region has been double-compressed with primary compression rate $q_{0}=60$. Middle and right: in the difference images $\Delta_{75}$ and $\Delta_{65}$ a "ghost" gradually appears as a darker region. Note also the noise in $\Delta_{75}$ and $\Delta_{65}$ due to the image texture.

$I_{q_{1}, q_{2}}$. The difference image $D_{q_{2}}$ of $I_{q_{1}}$ and $I_{q_{1}, q_{2}}$ is defined as

$$
D_{q_{2}}(x, y)=\frac{1}{3} \sum_{i \in\{R, G, B\}}\left(I_{q_{1}}(x, y, i)-I_{q_{2}}(x, y, i)\right)^{2}
$$

where $x$ and $y$ denote the pixel coordinates, and $i \in\{R, G, B\}$ the red, green and blue color channels.

If a region of the image has previously been compressed with a compression factor $q_{0}, q_{0}<q_{1}$, the squared differences become smaller for this part of the image as $q_{2}$ approaches $q_{0}$. This local region, termed "ghost", appears darker than the remaining image. This comes from the fact that if the coefficients of $q_{2}$ become more similar to the coefficients of (the unknown) $q_{0}$, similar artifacts are introduced in the image. For robustness to texture, $D_{q_{2}}(x, y)$ is averaged across small windows of size $w$. Thus, the differences are computed as

$\Delta_{q_{2}}(x, y)=\frac{1}{3 w^{2}} \sum_{i} \sum_{w_{x}=0}^{w-1} \sum_{w_{y}=0}^{w-1}\left(I_{q_{1}}\left(x+w_{x}, y+w_{y}, i\right)-I_{q_{2}}\left(x+w_{x}, y+w_{y}, i\right)\right)^{2}$

and normalized to lie in the range between 0 and 1 .

Fig. 1 shows an example of such a ghost. A rectangular double compressed region has been embedded with $q_{0}=60$ (left). Two difference images $\Delta_{75}$ and $\Delta_{65}$ are also shown. The resulting ghost can be clearly seen in $\Delta_{65}$. This can directly be forensically exploited by examining a number of difference images $\Delta_{q_{2}}$ for varying $q_{2}$. If a dark region appears, it is considered as doubly compressed.

However, in practice, the amount of human interaction is extremely timeconsuming for two reasons. First, one has to closely examine every image, as a ghost can be visually hard to distinguish from noise [2]. Second, the number of difference images can become very large: a ghost appears, if the JPEG grid of $\Delta_{q_{2}}$ is exactly aligned with the JPEG grid of the first compression using $q_{0}$. Thus, all possible 64 JPEG grid alignments must be visually examined. Ultimately, a human expert has to browse $64 \cdot|Q|$ images, where $|Q|$ is the number of difference images with $q_{2}<q_{1}$. In practice, there are often more than 300 difference images. 

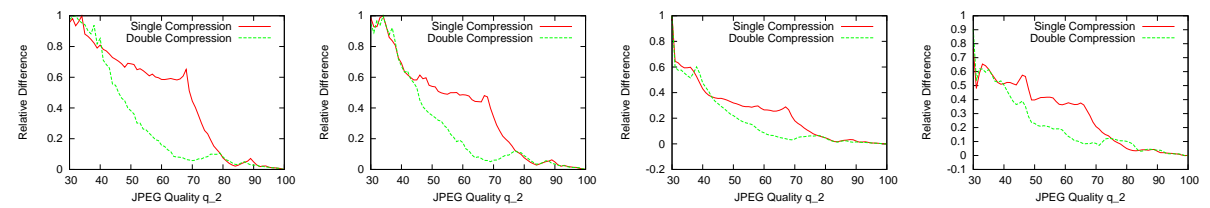

Fig. 2. Difference curves from example JPEG ROIs. The same ROI has been singleand double-compressed and is plotted in a joint diagram. In red, the difference curves for single compression are shown, in green for double compression.

\section{Feature Extraction}

The information about a JPEG ghost is contained in the differences of a single $w \times w$ window over different quality levels. Consider ROIs which have been single- or double-compressed. Example difference curves for this case are shown in Fig. 2. Here, the differences are computed over four different windows of an image with compression quality $q_{1}=84$, over 70 quality levels $30 \leq q_{2} \leq 100$ on a window size $w=16$. All differences are normalized between 0 and 1 . The red curves denote the differences for single compressed windows. The green curves show the same ROIs, but this time double compressed with $q_{0}=69$. The green curve exhibits a second minimum and rapid decay at (the unknown) $q_{0}$. Due to differences in image texture, this effect is stronger for the left graphs, than the right graphs.

Our method is based on the analysis of these difference curves. We estimate the quality level $q_{1}$ as the global minimum over the curves derived from all windows in the image. We then proceed as follows. Let $c(x)$ be the value of the difference curve for quality level $x$. We extracted six features that are defined on $c(x)$ for $30 \leq x \leq q_{1}$. Note that this range implies that we can not detect ghosts with $q_{0}<30$. However, this is mainly an engineering decision, as we considered cases of $q_{0}<30$ as very unlikely. Let, furthermore, $w_{1}(x)=(x-30) /\left(q_{1}-30\right)$ denote a weighting function that puts more emphasis on high JPEG qualities, and $w_{2}(x)=1-w_{1}(x)$ a weighting function that emphasizes low JPEG qualities. We employ the following features:

1. The weighted mean value of the curve,

$$
f_{1}=\frac{1}{\sum_{x=30}^{q_{1}} w_{1}(x)} \sum_{x=30}^{q_{1}} w_{1}(x) \cdot c(x) .
$$

2. The median of all values $c(x)$ for $30 \leq x \leq q_{1}$, i. e. $f_{2}=\mu_{1 / 2}$ where

$$
\left(P\left(c(x) \leq \mu_{1 / 2}\right) \geq \frac{1}{2}\right) \wedge\left(P\left(c(x) \geq \mu_{1 / 2}\right) \geq \frac{1}{2}\right),
$$

and $P(c(x) \leq x)$ denotes the cumulative distribution function of $c(x)$.

3. $f_{3}$ is the slope of the regression line through $c(x)$ for $30 \leq x \leq q_{1}$. 

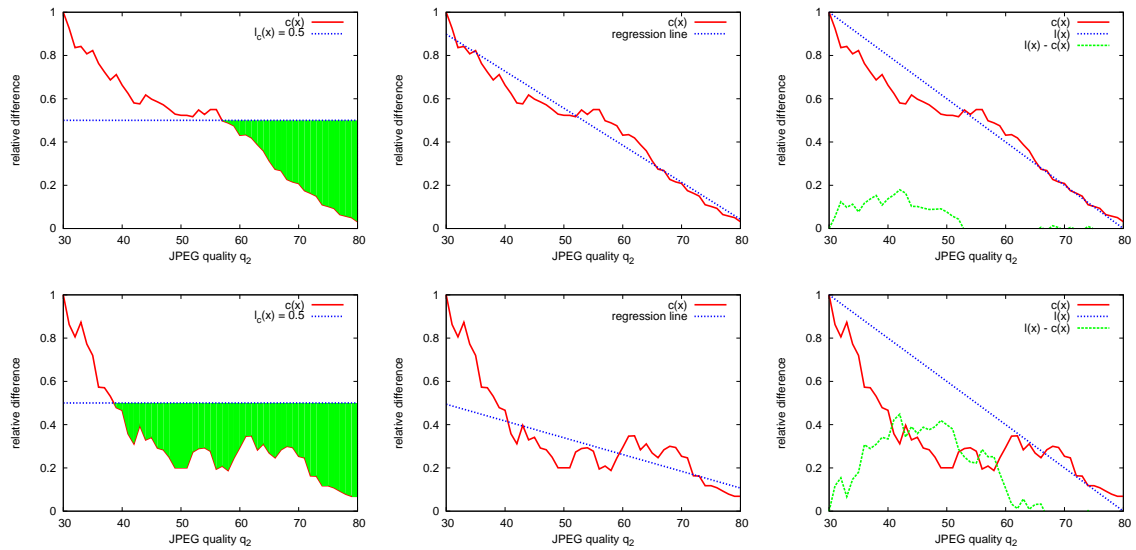

Fig. 3. Visualization of the proposed features. Top: a single-compressed window, bottom: a double-compressed window. Left: area below a normalized error of 0.5. Middle: regression line on the error curve. Right: squared distance of the curve to the line between $(30,1)$ and $\left(q_{1}, 0\right)$.

4. $f_{4}$ is the $y$-axis intercept of the regression line through $c(x)$ for $30 \leq x \leq q_{1}$.

5 . The weighted number of points of $c(x)$ with $c(x)<t=0.5$,

$$
f_{5}=\left(\sum_{x=30}^{q_{1}} w_{2}(x)\right)^{-1} \cdot \sum_{x=30}^{q_{1}} w_{2}(x) \cdot g_{5}(x), \text { where } g_{5}(x)= \begin{cases}1 & \text { if } c(x)<t \\ 0 & \text { otherwise }\end{cases}
$$

6. The average squared distance between the actual curve and the linear function $l(x)=1-(x-30) /\left(q_{1}-30\right)$, i. e. the line connecting $(30,1)$ and $\left(q_{1}\right.$, $0)$. More formally,

$$
f_{6}=\sum_{x=30}^{q_{1}} g_{6}(x), \text { where } g_{6}(x)= \begin{cases}(l(x)-c(x))^{2} & \text { if } l(x)>c(x) \\ 0 & \text { otherwise }\end{cases}
$$

The feature computation is well parallelizable, as every feature depends only on a spatially isolated window. The core idea of this feature set is to identify the steeper decay of the double-compression difference curve (see Fig. 2). Figure 3 illustrates the decision for the feature set on an example window. For each feature, we computed a histogram based on $2.5 \cdot 10^{6}$ image windows, see Fig. 4 . The compression quality levels of these windows were randomly chosen between 50 and 95 , with a fixed distance $q_{0}-q_{1}=20$. Feature values of single-compressed windows are plotted in green, while features of double-compressed windows are shown in red. From left to right, the distributions for $f_{1}$ to $f_{6}$ are shown. Although several blocks overlap within a feature, the majority of blocks exhibits good separation between single- and double-compression. 

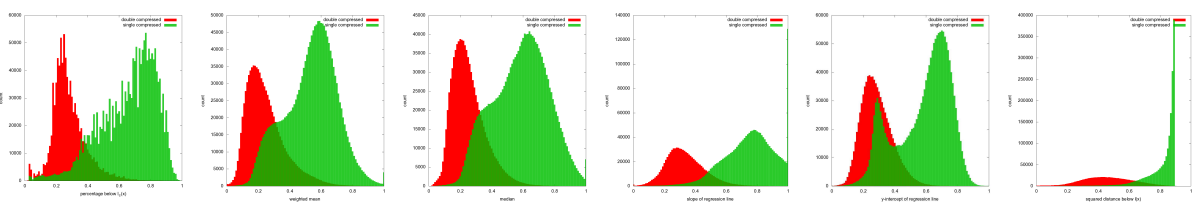

Fig. 4. Histograms of each of the six features, $f_{1}, f_{2}, \ldots, f_{6}$, shown from left to right. Red histograms are from double-compressed windows. Green ones correspond to singlecompressed windows.

\section{Classification}

Every block was classified separately, solely based on its features. We evaluated different classification algorithms: thresholding, Neural Networks, Random Forests, AdaBoost and Bayes. The openCV implementations of these algorithms were used. The values for thresholding were determined by computing the mean values of the feature distributions for single- and double-compressed areas. The actual threshold was then determined as the mean of means for each feature. A block was considered double-compressed, if at least three quarters of the feature values exceeded their respective threshold. The Neural Network was a Multilayer Perceptron with a 10-node hidden layer. The activation function is a sigmoid function with $\alpha=\beta=1$. For the Random Forests, we used 50 trees for classification, while for Discrete AdaBoost 100 trees. The cost functions of all classifiers were set to a balanced state of false positive and false negative rates.

\section{Experiments}

We used the Uncompressed Colour Image Database (UCID) [9] for evaluating our method. It consists of 1338 images of size $512 \times 384$. For each image, we created a single-compressed version, and two versions with single- and doublecompression, where a randomly chosen $192 \times 192$ pixels region is differently compressed than the background. In total, this yields twice as many single- as double-compressed pixels. Per image, we randomly selected $q_{1} \in[50 ; 95]$, and set $q_{0}=q_{1}-\delta$, where $5 \leq \delta \leq 20$. We varied the length $w$ of a window between 8 and 64 pixels. Following [5], we excluded very smooth image regions, i. e. windows with an intensity variance below 5 points. For the training of the classifiers, we used $10 \%$ of the images from all three classes. Note that the test- and training sets are still weakly correlated due to the high variability of the dataset.

As a quality measure, we used specificity and sensitivity. Let sc and dc denote "single-compressed" and "double-compressed", respectively. Then, let

$$
\mathrm{TP}=P(\mathrm{dc} \mid \mathrm{dc}), \quad \mathrm{TN}=P(\mathrm{sc} \mid \mathrm{sc}), \quad \mathrm{FP}=P(\mathrm{dc} \mid \mathrm{sc}), \quad \mathrm{FN}=P(\mathrm{sc} \mid \mathrm{dc})
$$

be the true positives, true negatives, false positives and false negatives, respectively. Then, specificity and sensitivity are defined as

$$
\text { specificity }=\frac{\mathrm{TN}}{\mathrm{TN}+\mathrm{FN}}, \quad \text { sensitivity }=\frac{\mathrm{TP}}{\mathrm{TP}+\mathrm{FN}} .
$$


Table 1. Experiments on the UCID database for shifted ghost detection on misaligned DCT grids at a per-window level.

\begin{tabular}{c|c||c|c|c|c|c}
$\delta$ & $w$ & Thresh. & MLP & RF & Boost. & Bayes \\
\hline 5 & 8 & $0.798 / 0.702$ & $0.866 / 0.826$ & $0.804 / 0.889$ & $0.834 / 0.886$ & $0.469 / 0.984$ \\
& 16 & $0.805 / 0.704$ & $0.855 / 0.880$ & $0.811 / 0.901$ & $0.841 / 0.893$ & $0.483 / 0.983$ \\
& 32 & $0.816 / 0.717$ & $0.865 / 0.891$ & $0.838 / 0.925$ & $0.847 / 0.919$ & $0.505 / 0.981$ \\
& 64 & $0.840 / 0.596$ & $0.897 / 0.833$ & $0.889 / 0.890$ & $0.907 / 0.870$ & $0.562 / 0.976$ \\
\hline 10 & 8 & $0.815 / 0.728$ & $0.865 / 0.864$ & $0.831 / 0.901$ & $0.852 / 0.896$ & $0.479 / 0.986$ \\
& 16 & $0.821 / 0.730$ & $0.869 / 0.873$ & $0.834 / 0.914$ & $0.858 / 0.910$ & $0.497 / 0.984$ \\
& 32 & $0.833 / 0.745$ & $0.835 / 0.935$ & $0.850 / 0.941$ & $0.865 / 0.934$ & $0.520 / 0.983$ \\
& 64 & $0.837 / 0.755$ & $0.917 / 0.847$ & $0.908 / 0.893$ & $0.924 / 0.869$ & $0.579 / 0.979$ \\
\hline 20 & 8 & $0.844 / 0.778$ & $0.888 / 0.910$ & $0.865 / 0.938$ & $0.895 / 0.934$ & $0.506 / 0.985$ \\
& 16 & $0.849 / 0.783$ & $0.895 / 0.906$ & $0.864 / 0.937$ & $0.902 / 0.938$ & $0.520 / 0.984$ \\
& 32 & $0.857 / 0.796$ & $0.865 / 0.935$ & $0.879 / 0.935$ & $0.912 / 0.957$ & $0.553 / 0.983$ \\
& 64 & $0.861 / 0.804$ & $0.925 / 0.892$ & $0.931 / 0.913$ & $0.938 / 0.916$ & $0.622 / 0.977$ \\
\hline
\end{tabular}

Note that two other popular measures, the false positive rate and the false negative rate, additively complement specificity and sensitivity to 1 . All results in the tables are presented as specificity/sensitivity pairs.

\subsection{Experiments on Individual Image Windows}

Tab. 1 shows the results for the evaluation per $w \times w$ window. Here, "Thresh.", "MLP", "RF", "Boost." and "Bayes" denote classification by thresholding, multilayer perceptron, random forests, discrete AdaBoost and the Bayesian classifier, respectively. The difference in the quality levels of primary and secondary compression is denoted as $\delta=q_{1}-q_{0}$. In order to have a relatively balanced number of single and double compressed pixels, we only evaluated the performance on the correct shift of the doubly-compressed region. However, when we evaluated the whole pipeline, we tested all 64 shifts of the JPEG grid.

The best performance per classifer (considering the sum of specificity and sensitivity) on all combinations of $\delta$ and the region size is printed in italics. As expected, typically the highest tested compression distance of $\delta=20$, together with the largest examined window size $64 \times 64$ yields best results. Note that AdaBoost performed best below the maximum windows size. Note also that for $\delta=10$ and $\delta=5$, boosting, followed by neural networks (MLP) and random forests (RF) all provide very strong results. Furthermore, the performance of these three methods degrades gracefully for smaller windows. Thus, as a preprocessing step for guiding a human expert towards a JPEG ghost location, we consider these three classifiers highly suitable. Additionally, the good discrimination for small values of $\delta$ improves over the results reported in [5], which reports $\delta \geq 20$ as a good quality distance for detection. In [7], detection rates for $\delta \leq 10$ vary between $50 \%$ and $70 \%$. 
Table 2. Experiments on the UCID database for ghost detection on aligned DCT grids at image level.

\begin{tabular}{c|c||c||c|c|c|c|c}
$\delta$ & $w$ & Lin et al. & Thresh. & MLP & RF & Boost. & Bayes \\
\hline 5 & 8 & $0.583 / 0.640$ & $0.806 / 0.766$ & $0.783 / 0.870$ & $0.756 / 0.929$ & $0.823 / 0.867$ & $0.576 / 0.846$ \\
& 16 & - & $0.816 / 0.760$ & $0.783 / 0.871$ & $0.762 / 0.934$ & $0.832 / 0.867$ & $0.646 / 0.841$ \\
& 32 & - & $0.812 / 0.755$ & $0.749 / 0.889$ & $0.726 / 0.949$ & $0.811 / 0.883$ & $0.742 / 0.822$ \\
& 64 & - & $0.830 / 0.481$ & $0.963 / 0.355$ & $0.978 / 0.375$ & $0.982 / 0.378$ & $0.882 / 0.606$ \\
\hline 10 & 8 & $0.658 / 0.597$ & $0.820 / 0.759$ & $0.774 / 0.874$ & $0.913 / 0.857$ & $0.918 / 0.880$ & $0.594 / 0.892$ \\
& 16 & - & $0.827 / 0.758$ & $0.777 / 0.870$ & $0.897 / 0.873$ & $0.916 / 0.899$ & $0.772 / 0.815$ \\
& 32 & - & $0.830 / 0.737$ & $0.776 / 0.857$ & $0.882 / 0.883$ & $0.916 / 0.895$ & $0.840 / 0.845$ \\
& 64 & - & $0.852 / 0.439$ & $0.946 / 0.377$ & $0.968 / 0.416$ & $0.989 / 0.412$ & $0.938 / 0.684$ \\
\hline 20 & 8 & $0.705 / 0.605$ & $0.832 / 0.880$ & $0.864 / 0.941$ & $0.905 / 0.960$ & $0.997 / 0.939$ & $0.533 / 0.997$ \\
& 16 & - & $0.858 / 0.883$ & $0.892 / 0.957$ & $0.904 / 0.973$ & $0.996 / 0.956$ & $0.647 / 0.997$ \\
& 32 & - & $0.867 / 0.868$ & $0.865 / 0.947$ & $0.908 / 0.972$ & $0.993 / 0.960$ & $0.839 / 0.988$ \\
& 64 & - & $0.933 / 0.484$ & $0.866 / 0.517$ & $0.959 / 0.519$ & $0.997 / 0.507$ & $0.960 / 0.955$ \\
\hline
\end{tabular}

\subsection{Experiments on Automated Tampered Image Detection}

For comparison to other methods, we implemented a straightforward tampered image classifier based on the recognition of partially double-compressed JPEG images. To remove outliers on the marked windows from the previous section, we applied a $3 \times 3$ pixels morphological opening with a cross-topology on these markings. We considered an image tampered, if $10 \%$ of the windows are marked. Note that an embedded foreground-ghost contains about $20 \%$ double-compressed pixels, a background-ghost about $100 \%-20 \%=80 \%$. As before, we created three images from every UCID image. Once completely single-compressed, once with an embedded foreground-ghost, and once with an embedded background-ghost. Tab. 2 shows the result for JPEG ghosts that were exactly aligned with the JPEG grid. We used the same notation as in the previous Subsection.

For comparison, we evaluated the method of Lin et al. [7] on our test set. As this method operates on $8 \times 8$ windows, only these results are presented. The approach of Lin et al. is more general, in the sense that it can also detect double-compression where $q_{0}>q_{1}$. However, this comes at the expense of the accuracy in the presence of very small differences in the compression parameters. Thus, if the initial assumption $q_{0}<q_{1}$ for JPEG ghosts is fulfilled, the proposed method provides much higher specificity and sensitivity rates.

The best success rates do not occur at the larger window sizes. This is due to the fact that the embedded foreground ghosts of $192 \times 192$ pixels are comparably small. When applying morphological opening on the windows that have been marked as double-compressed, more accurate detectors lose too many windows on the boundary of the marked region. This renders very large window sizes less successful. One notable exception is the Bayesian classifier. As can be seen from Tab. 1, Bayesian classification exhibits very low specificity, i. e. creates many erroneously marked regions. The morphological operator removes a large 
Table 3. Experiments on UCID database for shifted ghost detection on misaligned DCT grids on image level.

\begin{tabular}{c|c||c|c|c|c|c}
$\delta$ & $w$ & Thresh. & MLP & RF & Boost. & Bayes \\
\hline 5 & 8 & $0.742 / 0.772$ & $0.982 / 0.904$ & $0.923 / 0.960$ & $0.990 / 0.955$ & $0.907 / 0.981$ \\
& 16 & $0.755 / 0.708$ & $0.993 / 0.934$ & $0.940 / 0.952$ & $0.987 / 0.944$ & $0.919 / 0.982$ \\
& 32 & $0.750 / 0.698$ & $0.973 / 0.915$ & $0.969 / 0.952$ & $0.984 / 0.947$ & $0.923 / 0.983$ \\
& 64 & $0.763 / 0.468$ & $0.967 / 0.765$ & $0.992 / 0.843$ & $1.000 / 0.809$ & $0.951 / 0.983$ \\
\hline 10 & 8 & $0.762 / 0.794$ & $0.978 / 0.932$ & $0.938 / 0.957$ & $0.978 / 0.951$ & $0.923 / 0.983$ \\
& 16 & $0.779 / 0.733$ & $0.975 / 0.913$ & $0.955 / 0.954$ & $0.984 / 0.948$ & $0.945 / 0.983$ \\
& 32 & $0.791 / 0.728$ & $0.981 / 0.968$ & $0.961 / 0.957$ & $0.986 / 0.950$ & $0.951 / 0.983$ \\
& 64 & $0.795 / 0.646$ & $0.984 / 0.755$ & $0.993 / 0.803$ & $0.998 / 0.775$ & $0.966 / 0.986$ \\
\hline 20 & 8 & $0.784 / 0.836$ & $0.977 / 0.955$ & $0.969 / 0.972$ & $0.993 / 0.971$ & $0.987 / 0.985$ \\
& 16 & $0.802 / 0.795$ & $0.963 / 0.939$ & $0.978 / 0.972$ & $0.995 / 0.969$ & $0.993 / 0.987$ \\
& 32 & $0.806 / 0.786$ & $0.862 / 0.953$ & $0.948 / 0.963$ & $0.995 / 0.971$ & $0.995 / 0.988$ \\
& 64 & $0.810 / 0.659$ & $0.988 / 0.833$ & $0.994 / 0.806$ & $0.999 / 0.818$ & $0.997 / 0.988$ \\
\hline
\end{tabular}
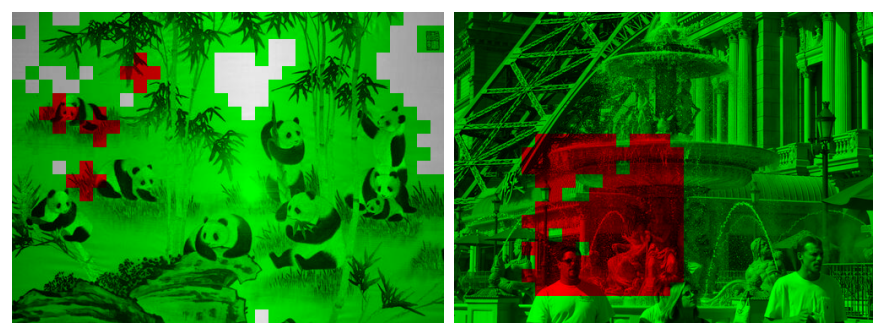

Fig. 5. Two example markings on individual windows. Green and red are single- and double compressed, respectively. Gray denotes low contrast regions. Left: the rectangular double compression region could only in the high-contrast windows be recovered. Right: the double compression region is clearly visible. In a classification on image level, the left example is a false negative case, the right example true positive.

number of these false positive markings, and makes detection with larger window sizes possible. In comparison, the remaining classifiers exhibit their peak performance at window sizes around $16 \times 16$ pixels. Again, discrete Adaboost clearly outperforms the other methods.

In shifted double-compression, the grid of the inserted region is not required to properly align with the JPEG grid of the background. To detect such tampered images, we computed all 64 shifts and selected the one with the highest response of double-compressed blocks (see Tab. 3 for the results). A surprising result is that shifted double JPEG compression can be slightly better discriminated than the non-shifted version: while the overall best result in Tab. 2 is $0.997 / 0.939$, several results in Tab. 3 perform better, e. g. the Bayesian classifier on a $64 \times 64$ grid for $\delta=20$ with $0.997 / 0.988$. During shifted double-compression, $q_{1}=100$ is often (wrongly) estimated. Interestingly, the increased gap between $q_{0}$ and $q_{1}$ improves classification. Based on our results, we recommend the use of AdaBoost 
for JPEG ghost detection. Its reliability on small window sizes yields in many cases a high level of detail in the marked blocks (see Fig. 5). The quality difference $\delta$ can be decreased to an empirical minimum of 5 points. In such cases, other methods often exhibit difficulties; e. g. [5] and [7] reported increased error rates for $\delta<20$ and $\delta<10$, respectively.

\section{Conclusions}

We proposed a JPEG-based forensic algorithm to automatically distinguish single-compressed and double-compressed image regions. We presented a classification scheme that exploits the JPEG-ghost effect, but completely removes the requirement of browsing the images. Best results were achieved by training a boosted classifier on 6 specially designed features. The classification performance is encouraging. The best specificity and sensitivity are 0.912 and 0.957 , respectively. For tampering detection, the difference in primary and secondary compression may be as small as $\delta=5$.

\section{References}

1. Barni, M., Costanzo, A., Sabatini, L.: Identification of Cut \& Paste Tampering by Means of Double-JPEG Detection and Image Segmentation. In: International Symposium on Circuits and Systems. pp. 1687-1690 (May 2010)

2. Battiato, S., Messina, G.: Digital Forgery Estimation into DCT Domain - A Critical Analysis. In: Multimedia in Forensics, Security and Intelligence. pp. 37-42 (Oct 2009)

3. Bayram, S., Sencar, H.T., Memon, N.: A survey of copy-move forgery detection techniques. In: IEEE Western New York Image Processing Workshop (2009)

4. Farid, H.: A Survey of Image Forgery Detection. Signal Processing Magazine 26(2), 16-25 (Mar 2009)

5. Farid, H.: Exposing Digital Forgeries from JPEG Ghosts. IEEE Transactions on Information Forensics and Security 1(4), 154-160 (2009)

6. Huang, F., Huang, J., Shi, Y.Q.: Detecting Double JPEG Compression With the Same Quantization Matrix. IEEE Transactions on Information Forensics and Security 5(4), 848-856 (Dec 2010)

7. Lin, Z., He, J., Tang, X., Tang, C.K.: Fast, Automatic and Fine-grained Tampered JPEG Image Detection via DCT Coefficient Analysis. Pattern Recognition 52(11), 2492-2501 (Nov 2009)

8. Qu, Z., Luo, W., Huang, J.: A Convolutive Mixing Model for Shifted Double JPEG Compression with Application to Passive Image Authentication. In: International Conference on Acoustics, Speech and Signal Processing. pp. 1661-1664 (Mar 2008)

9. Schaefer, G., Stich, M.: UCID - An Uncompressed Colour Image Database. In: SPIE Storage and Retrieval Methods and Applications for Multimedia. pp. 472 480 (Jan 2004)

10. Sencar, H., Memon, N.: Overview of State-of-the-art in Digital Image Forensics. Algorithms, Architectures and Information Systems Security pp. 325-344 (2008) 